CHÁVEZ-ZÚÑIGA, Pablo; SOTO-LARA, José Julián. Mortalidad infantil en Santiago: representaciones y discursos, Chile, 1860-1914. História, Ciências, Saúde - Manguinhos, Rio de Janeiro, v.25, n.4, out.-dez. 2018, p.1281-1300.

\section{Mortalidad infantil en} Santiago: representaciones y discursos, Chile, 1860-1914

\section{Infant mortality in Santiago: representations and discourses, Chile, 1860-1914}

\author{
Pablo Chávez-Zúñiga \\ Doctor en Historia, Facultad de Filosofía y Humanidades/ \\ Universidad de Chile. \\ Santiago - Provincia de Santiago - Chile \\ pablo.chavez.zuniga@gmail.com \\ José Julián Soto-Lara \\ Investigador asociado, Centro de Estudios Históricos/ \\ Universidad Bernardo O'Higgins. \\ Santiago - Provincia de Santiago - Chile \\ jose.julian.soto@gmail.com
}

Recebido em 29 mar. 2017.

Aprovado em 24 out. 2017.

\section{Resumen}

Se exploran las características de la mortalidad infantil en Santiago durante una fase histórica en que los indicadores demográficos alcanzaron cifras altísimas transformándose el fenómeno en un problema médico y político. Luego de comentar algunas investigaciones historiográficas sobre la temática, se demuestra cuantitativamente el comportamiento de la población infantil, recurriendo a fuentes primarias - de la medicina, la prensa y el Registro Civil - y bibliografía. Posteriormente, se describen las causas que las elites esgrimieron para explicar las muertes infantiles: materiales, morales y culturales. Las conclusiones abordan la promulgación de la ley de la infancia desvalida, los inicios de un conocimiento sobre la salud de los niños y la inauguración del Hospital Infantil.

Palabras clave: mortalidad infantil; Santiago de Chile; medicina; política; prensa.

\section{Abstract}

The article explores the characteristics of infant mortality in Santiago during a historical period in which the demographic indicators reached very high figures turning the phenomenon into a medical and political issue. After commenting on historiographic data, it demonstrates the behavior of the infant population quantitatively, taking into account primary sources - from medicine, the press and civil records - and bibliography. It also exposes the motives employed by the elite to justify the children's deaths: materially, morally and culturally. The conclusion addresses the promulgation of the defenseless child law, the beginnings of knowledge on child health and the inauguration of the Child Hospital.

Keywords: infant mortality; Santiago de Chile; medicine; politics; press. 
$\mathrm{L}$ a catástrofe demográfica de la mortalidad infantil santiaguina, en torno al cambio de siglo XIX-XX, se expresó estadísticamente con un promedio de veinte niños muertos al día. ${ }^{1}$ De un total de 18.300 defunciones en 1901 - escribió el pediatra Luis Calvo Mackenna (1919, p.25) - 11.401 fueron niños. El fenómeno fue uno de los principales hitos de la denominada "cuestión social" chilena, producida por el crecimiento poblacional en la ciudad y la displicencia de la clase política para mejorar las condiciones de vida de los campesinos urbanizados (Salazar, 1985; Illanes, 1993; Grez Toso, 1997; Romero, 1984, 2007; Torres, 1986, 2011).

Durante este período, la elite médica elaboró un discurso científico y moral sobre la mortalidad donde los niños pobres portaban "naturalmente" los males sociales. El mismo razonamiento se aplicó al estudiar el modus vivendi de los procreadores. En el plano político, el Ministerio del Interior y el Consejo Superior de Higiene Pública adolecieron de lentitud en la acción médica, acusando deficiencias legislativas. Sin superar un enfoque preventivo, actuaron y discutieron una vez desarrolladas las desgracias sanitarias. Consecuencias de ese estilo de planificación fueron los indicadores "escandalosos" de la mortalidad infantil.

Estudiar el impacto del fenómeno en Santiago no fue una decisión azarosa. El límite inicial, la década de 1860, representa los inicios de la conformación de un conocimiento médico sobre la infancia, lo que permite comprender el trazo complejo, discontinuo, resistente y receptor de cambios culturales-higiénicos-históricos. Por otro lado, el cambio de siglo coincidió con una preocupación legislativa mayor: legislación sobre la vivienda obrera (1906) y la infancia desvalida (1912). Esto fue apoyado por la medicina con la inauguración del Hospital Infantil en 1914.

La elección de Santiago para el estudio historiográfico se basó en la elevadísima tasa de mortalidad infantil, comparada con otras ciudades; ${ }^{2}$ en su centralidad administrativa, desde donde se planificaron las políticas sanitarias para esta ciudad y el resto del país; y, por último, en la ubicación de las instituciones científicas, lugares de discusión de la elite médica de Chile. ${ }^{3}$

Los estudios europeos sobre el fenómeno se han interesado por el cuidado físico infantil durante la medicalización, iniciado en la segunda mitad del siglo XIX. La reducción de las enfermedades infecciosas y la urbanización disminuyó la mortalidad en edades tempranas (Lebrun, 1982; Masuy-Stroobant, 1983; Belletini, 1983; Apple, 1987; Ruggiero, 1992; Ballester, Balanguer, 1995; Arbaiza, Guerrero, Pareja, 1996; Apple, Golden, 1997; Fariñas, Sanz Gimeno, 1999; Sanz Gimeno, Fariñas, 2002; Morata, 2003; Ballester, 2005). La argumentación de diagnósticos y tratamientos pediátricos era dificultosa, porque esa ciencia recién comenzaba su institución. En la práctica, sus conocimientos coexistieron con otros de carácter popular (Bernabeu, López, 1987; Bernabeu, 1993; Rodríguez, 1998, 2003; Bernabeu et al., 2003; Revuelta, 2012, 2013, 2015).

En América Latina existe una producción bibliográfica considerable sobre la mortalidad infantil (García, 2016). En Colombia, la medicalización infantil se desarrolló en dos procesos simultáneos: la circulación-apropiación del conocimiento europeo, y el sustrato local de la población analizada (García, 1999; Gallo, Márquez, 2011). En Uruguay, país con la tasa de mortalidad infantil más baja de la región, esos índices se estancaron a inicios del siglo XX produciendo debates pediátricos y políticos. Décadas después, en 1930, el Estado de 
Bienestar priorizó la salud de los lactantes favoreciendo una nueva disminución de los índices comentados (Birn, 2003, 2007, 2008).

En Chile, Eduardo Cavieres Figueroa (2001) demostró para el caso de Valparaíso entre 1890-1950 la modificación de los sentimientos y cuidados maternos hacia los hijos en momentos de enfermedad. Demográficamente, sostuvo que los procesos económicos, culturales y médicos no disminuyeron la mortalidad elevada, situación que avanzado el siglo mejoró, reduciendo las cifras mortales. Por su parte, Gabriel Salazar (2006) investigó la precarización de las economías familiares, situación favorable al abandono. Basándose en datos cuantitativos demostró la proporción alta de niños en relación al total de habitantes del país; el aumento porcentual de niños ilegítimos respecto al número anual de nacidos entre 1848 y 1916; y la permanencia de la mortalidad infantil dentro y fuera de los recintos protectores de la infancia. Además, metodológicamente rescató las "voces" y prácticas de la niñez. En una línea similar, María Angélica Illanes (1991) y Jorge Rojas Flores (2006, 2010) aportaron una mirada histórico social a la experiencia de ser niño en los inicios del siglo pasado.

Andrés Estefane (2004, 2012, 2016), al estudiar la conformación del sistema estadístico chileno, reflexionó sobre la instalación de las redes estatales en el territorio a través de la Oficina de Estadística. Dio cuenta de las dificultades de los párrocos para efectuar registros demográficos confiables. Entre esas señaló las omisiones de datos por creencias religiosas; la exclusión en los registros de los neonatos nacidos muertos por no estar bautizados; y los envíos a instituciones erróneas de los padrones demográficos. Estefane destacó las dificultades y los engranajes en la construcción estatal de las cifras: la delimitación de las provincias, el entramado entre las regiones estatales y las circunscripciones parroquiales, la preparación de funcionarios para recolección de padrones fiables y la uniformidad de criterios para establecer causas de muertes. Dichos procesos tardaron décadas en materializarse y dimensionar cuantitativamente el problema de la mortalidad infantil en el país.

La reconstrucción histórica de los infantes está abordada por la constitución familiar, las características de las viviendas urbanas y rurales, los ambientes propicios para la enfermedad y las circunstancias que incidieron en sus abandonos (Salinas, Delgado, 1990; Salinas, 1991; Delgado, 2001; Goicovic, 2005). De esa manera, la mortalidad infantil estuvo anclada en las experiencias de niños huérfanos y su tránsito en instituciones benéficas. Las elevadas cifras de niños muertos generaron discusiones entre los médicos, sobre las condiciones de los ingresados y los cuidados que demostraban los antecedentes de una medicina enfocada en la infancia (Milanich, 1998, 2001, 2004, 2009, 2011; Palma, 2009; Osta, 2016).

El poder político, mediante leyes, normativas y ordenanzas construyó una visión de la enfermedad a través de clasificaciones, diagnósticos y tratamientos médicos (Sater, 2003). Ante la visión clásica sobre tal extensión del ámbito sanitario, la historiografía ha colocado la mirada en las resistencias cotidianas de los pacientes y los procesos que destacan la aplicación de estos proyectos (Armus, 2001, 2002, 2009). Esta perspectiva se extiende hacia las condiciones de la ciudad que albergó a los infantes, a inicios del siglo $\mathrm{XX}$ : acequias, hacinamiento, viviendas precarias, todas causales de la alta mortalidad (Moreno, 2000). ${ }^{4}$ 
Varios estudios han elaborado la temática de las modificaciones en la alimentación infantil y los significados de la maternidad (Zárate, 2005, 2007, 2010, 2011; Ehrick, 2001; Pita, 2004; Rustoyburu, 2012, 2015, 2016). La preocupación de los facultativos por los altos índices de mortalidad infantil promovió los debates sobre la nutrición de los párvulos, ya que una alta proporción de las muertes fue atribuido a la mala calidad de la leche (descomposición, sobre todo en verano), la ausencia de higiene en la manipulación, carencia de regulación sanitaria en la venta de lácteos y despreocupación porque a los recién nacidos les suministraban comidas que no correspondían a su edad (carne, ulpo, pescados, caldos, entre otros). En ese contexto, los médicos establecieron fórmulas científicas legitimando su labor disciplinaria, disminuyendo las cifras de fallecimiento por esta causa. En Brasil, María Martha de Luna Freire $(2008,2010)$ discute el cuerpo femenino como una construcción cultural con significados de amor, enfermedad y maternidad.

De acuerdo con las tesis anteriores, esta investigación pretende responder cuestiones que requieren argumentarse con mayores evidencias históricas: ¿Cuáles fueron las causas de la alta mortalidad infantil? ¿Qué factores explican la mirada hacia el problema y la relación entre los médicos y los sectores populares? ¿La tasa de mortalidad disminuyó a causa de las prescripciones médicas?

Por eso, el ejercicio heurístico consideró tres tipos de registros. Por un lado, las revistas médicas donde se discutió científicamente sobre la mortalidad infantil: Médico Práctico, Médica de Chile, Chilena de Higiene y Beneficencia Pública. Por otro, se revisaron memorias, actas de congresos y otros estudios ocupados de la temática. Finalmente se recogieron datos y opiniones publicadas por periódicos santiaguinos, los que frecuentemente reprodujeron las fuentes señaladas (El Mercurio, Las Últimas Noticias, El Ferrocarril, La Época, Diario Popular y El Chileno). Cada uno de los periódicos nombrados tuvo una singularidad ideológica que determinó sus sesgos editoriales.

\section{La muerte desde las cifras}

Durante el siglo XIX y parte del XX, las estadísticas de la mortalidad infantil causaron problemas en las instituciones responsables de establecerlas: la Iglesia católica y, desde 1884, el Registro Civil. ${ }^{5}$ En efecto, su disparidad provocó diferentes interpretaciones sobre el fenómeno y cómo solucionarlo. ${ }^{6}$ Médicos, políticos y periodistas reconocieron la gravedad del hecho en el país que lo situaba entre uno de los más afectados en el mundo.

"Chile un cementerio", "Estadística desconsoladora" o "La India en Chile" fueron algunos titulares grandilocuentes que reprodujeron la magnitud de la mortalidad infantil. Otras noticias, como la titulada "Ocupamos un lugar muy superior de las ciudades que tienen mayor mortalidad en el orbe entero" (Mortalidad, 7 ene. 1905, p.8), estaban respaldadas con datos de la Oficina Central de Estadísticas y comparaba los indicadores capitalinos con los de otras metrópolis. ${ }^{7}$

El doctor Adolfo Murillo miró con recelo las ciudades transformadas, metafóricamente, en cementerios. Era partidario de utilizar "el grueso escalpelo del anatomista, ya que no se necesitan disecciones prolijas, porque la llaga es grande y asquerosa" (Murillo, 1896, p.9). Heredero del paradigma "atmosférico", no comprendía cómo Santiago, con su buen clima 
y ubicación, compartía cifras con ciudades africanas y asiáticas. ${ }^{8}$ En el mismo sentido, la médica Eloísa Díaz (1906) comparaba la mortalidad chilena con la de los países epidémicos y más insalubres del planeta.

La mortalidad infantil en proporción a la mortalidad general en el decenio 1858-1868 fue de 57,6\% ascendiendo entre 1869-1879 a un 62,5\% (La mortalidad..., mar. 1875, p.340). Con posterioridad, el médico Carlos Sommers (1896, p.373) publicó los porcentajes de párvulos muertos en 1892, 1893, 1894 y 1895 que fueron, respectivamente, 52, 53, 54 y 49 por ciento.

Otros datos mostraron un porcentaje también alto de la mortalidad infantil en relación a la mortalidad general (entre un 65 y 70\%), es decir, siete de cada diez muertos fueron niños entre 1860-1863 (Tabla 1). Durante esos años la población santiaguina aumentó continuamente y con eso la mortalidad. El crecimiento natural poblacional se detuvo, comportamiento típico de los regímenes demográficos tradicionales (Chaunu, 1982; Lindón, Hiernaux, 2006; Sánchez, 2008).

Tabla 1: Relación entre mortalidad general e infantil (menores de 7 años) en la Provincia de Santiago $(1860-1883)^{9}$

\begin{tabular}{|c|c|c|c|c|c|}
\hline Año & Población general & $\begin{array}{c}\text { Población menor } \\
\text { de } 7 \text { años }\end{array}$ & $\begin{array}{c}\text { Mortalidad } \\
\text { general }\end{array}$ & Mortalidad infantil & Proporción \\
\hline 1860 & 159.968 & 27.514 & 6.452 & 4.822 & $75 \%$ \\
\hline 1861 & 161.908 & 27.848 & 6.793 & 4.357 & $64 \%$ \\
\hline 1862 & 163.300 & 28.187 & 5.680 & 4.393 & $77 \%$ \\
\hline 1863 & 165.312 & 28.433 & 6.581 & 5.153 & $78 \%$ \\
\hline 1864 & 166.571 & 28.650 & 6.828 & 5.245 & $77 \%$ \\
\hline 1865 & 168.462 & 28.975 & 6.452 & 4.974 & $77 \%$ \\
\hline 1866 & 170.247 & 29.282 & 5.852 & 4.513 & $77 \%$ \\
\hline 1867 & 173.093 & 29.772 & 5.702 & 4.395 & $77 \%$ \\
\hline 1868 & 175.916 & 30.257 & 5.262 & 3.832 & $73 \%$ \\
\hline 1869 & 179.562 & 30.884 & 7.438 & 5.872 & $79 \%$ \\
\hline 1870 & 180.992 & 31.130 & 5.724 & 3.972 & $69 \%$ \\
\hline 1871 & 184.753 & 31.777 & 6.201 & 4.531 & $73 \%$ \\
\hline 1872 & 188.062 & 32.346 & 7.339 & 5.186 & $71 \%$ \\
\hline 1873 & 190.086 & 32.694 & 6.943 & 5.027 & $72 \%$ \\
\hline 1874 & 193.197 & 33.230 & 6.658 & 4.450 & $67 \%$ \\
\hline 1875 & 195.967 & 33.706 & 7.324 & 5.151 & $70 \%$ \\
\hline 1876 & 198.014 & 34.058 & 8.316 & 5.731 & $69 \%$ \\
\hline 1877 & 199.215 & 34.265 & 6.822 & 4.803 & $70 \%$ \\
\hline 1878 & 201.592 & 34.674 & 7.709 & 5.163 & $67 \%$ \\
\hline 1879 & 203.073 & 34.928 & 7.299 & 4.987 & $68 \%$ \\
\hline 1880 & 205.498 & 35.345 & 8.601 & 6.273 & $73 \%$ \\
\hline 1881 & 206.512 & 35.520 & 6.744 & 4.777 & $71 \%$ \\
\hline 1882 & 210.115 & 36.140 & 7.314 & 5.150 & $70 \%$ \\
\hline 1883 & 212.829 & 36.606 & 7.256 & 5.739 & $79 \%$ \\
\hline
\end{tabular}

Fuente: tabla elaborada a partir de los registros parroquiales sistematizados por el doctor Ricardo Dávila Boza (1899, p.277-294). 
Entre 1856 y 1904 el alto índice de niños nacidos y muertos fue una tendencia. Los fallecidos superaron a hombres y mujeres siendo casi la mitad del total de las muertes mensuales (Gráfico 1). La serie muestra cotas bianuales altas seguidas por descensos. Algunas de esas cotas se explican por la ocurrencia de brotes epidemiológicos violentos, por ejemplo, alfombrilla (1866), cólera (1866) y peste bubónica (1904-1907), todas ocurridas en medio de las enfermedades endémicas (viruela, tisis, fiebres). Entre abril y septiembre la mortalidad infantil tendió a disminuir; entre octubre y marzo mostró signos de aumento, según los médicos, a causa de las enfermedades estomacales estivales. Éstas, a diferencia de las respiratorias invernales, cobraron mayor número de víctimas. Es probable que la cota de noviembre de 1900, superior a los 1.800 fallecimientos, a pesar de un brote de alfombrilla, fuese producida por un error tipográfico del Registro Civil.

Gráfico 1: Mortalidad mensual en el Departamento de Santiago (hombres, mujeres y niños, ene. 1856-nov. 1904)

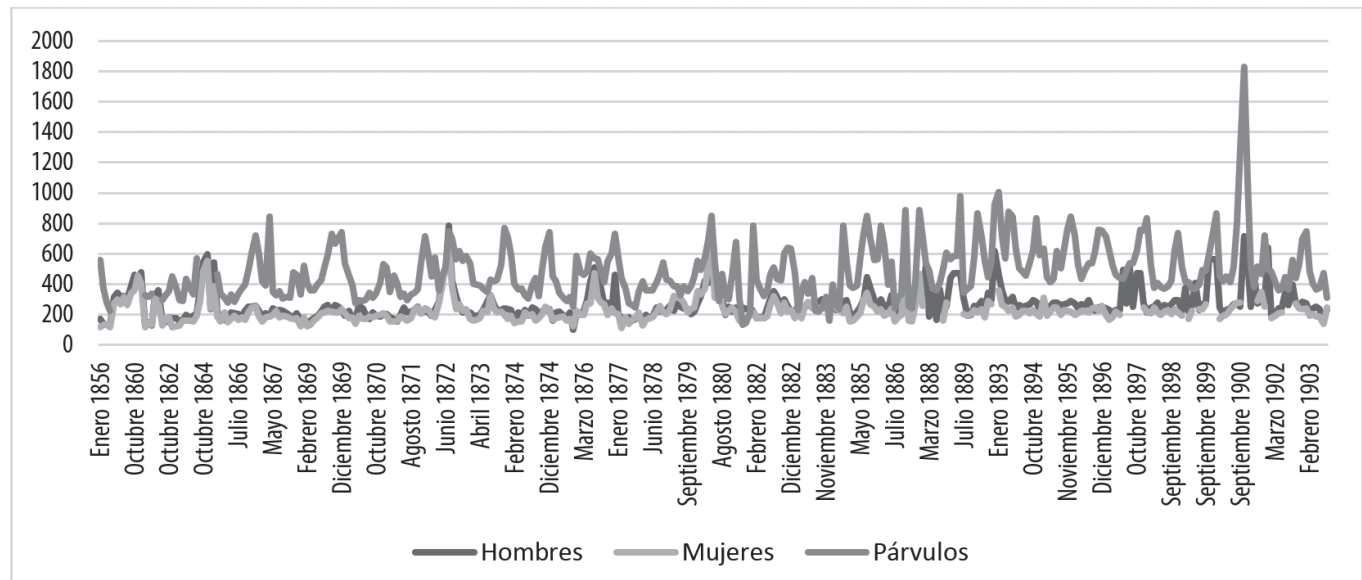

Fuente: elaboración de los autores basada en los cuadros mensuales publicados en la prensa de la época (principalmente El Ferrocarril) con datos de las parroquias (hasta 1883) y del Registro Civil (desde 1884).

El Boletín de Higiene y Demografía fue una publicación médica que entregaba una estadística mensual sobre causas de la mortalidad, su impacto etario diferencial y el origen legítimo o ilegítimo del nacimiento de los difuntos. De acuerdo con el Boletín se puede sostener que la mortalidad de niños menores de un año en relación al total fue constante durante los primeros años del siglo veinte, bordeando siempre el tercio (Tabla 2). Hipotéticamente, sumadas a esas cifras los muertos entre uno y siete años, el resultado sería similar al expuesto en la tabla anterior con porcentajes de muerte cercanos al 70\%.

Tabla 2: Relación entre la mortalidad general y defunciones de menores de un año

\begin{tabular}{cccc}
\hline Año & Mortalidad general & Mortalidad hasta 1 año & Porcentaje \\
\hline 1898 & 11.639 & 3.818 & $33 \%$ \\
1899 & 11.551 & 3.969 & $34 \%$ \\
1900 & 15.950 & 6.087 & $38 \%$ \\
1901 & 11.534 & 3.978 & $35 \%$
\end{tabular}




\begin{tabular}{llll}
1902 & 10.358 & 3.492 & $34 \%$ \\
1903 & 10.035 & 3.536 & $35 \%$ \\
1904 & 10.965 & 3.562 & $33 \%$ \\
1905 & 13.751 & 4.425 & $32 \%$ \\
1906 & 12.142 & 3.746 & $31 \%$ \\
1907 & 11.285 & 3.936 & $35 \%$ \\
1908 & 13.074 & 4.902 & $38 \%$ \\
1909 & 17.773 & 4.636 & $26 \%$ \\
1910 & 16.262 & 4.528 & $28 \%$ \\
1911 & 13.551 & 4.452 & $33 \%$ \\
\hline
\end{tabular}

Fuente: elaboración de los autores a partir de los datos mensuales entregados en Del Río (1898-1911).

Hasta la creación del Registro Civil, los párrocos enviaban mensualmente a las intendencias un informe demográfico que el oficial de estadística trasladaba a la Oficina Central de Estadística. En ocasiones lo olvidaban impidiendo "formar un cómputo exacto del movimiento de población en nuestro departamento" (Movimiento..., 7 jul. 1856, p.3), produciendo una discontinuidad numérica de los fallecidos. Las publicaciones evidenciaron esas faltas dificultando una panorámica del fenómeno, máxime porque en los curatos "la estadística deja mucho que desear" (Mortalidad..., 18 dic. 1882, p.2). Los curas - sobre todo rurales - debido a sus múltiples obligaciones administrativas descuidaban las anotaciones de los nacimientos y muertes. A ello se sumó la práctica del abandono del cuerpo muerto impidiendo la producción de documentación válida sobre el difunto.

El Registro Civil no superó esos problemas, debido a que un porcentaje importante de nacidos no fue inscrito en la institución (Chile..., 18 sep. 1908, p.7). Otra cosa sucedió con las muertes, que necesariamente debían inscribirse para inhumar los cuerpos. De ese modo, el número de defunciones tuvo una cierta correlación con la realidad. El doctor Adolfo Murillo (1896, p.7) señaló: “las cifras de mortalidad tan desconsoladoras, no están distantes de la verdad; porque recurriendo a los antiguos anuarios, cuando el registro se llevaba por parroquias y no por circunscripciones, siempre la mortalidad urbana fue considerable".

Los trámites de las sepultaciones infantiles en el Registro Civil debían contar con dos testigos de la muerte (Anguita, 1913; Chile, 16 jul. 1884). La ausencia, entre éstos, de un médico que comprobase la defunción suscitó críticas. El médico Emilio Croizet (1913, p.5) juzgó: "no podemos establecer un tanto por ciento más o menos preciso, por cuanto los informes obtenidos por nuestra Oficina Central de Estadística son sumamente incompletos, ya que en miles de casos viene la declaración de muerte sin informe médico y solamente con dos testigos tal como lo permite la ley".

Por esas razones, la estadística padeció errores que desconocían científicamente las causas de las muertes. Para mejorarla, el médico Alejandro del Río propuso la institución de médicos comprobadores de defunciones en Santiago y Valparaíso con lo cual posibilitaba "no solo precisar las causas de muerte y edad de los fallecidos bajo un criterio fijo y uniforme, sino hacer caer la sanción de la lei sobre las personas que no hubieran cumplido, respecto de los 
fallecidos, la obligación de inscribir los nacimientos dentro de los treinta días siguientes a su ocurrencia" (La mortandad..., 29 dic. 1902, p.6).

Esta medida tuvo gran importancia. En el plano jurídico permitió iniciar investigaciones para descubrir homicidios e infanticidios, así mismo permitió la constitución de la medicina legal. En lo político, la demografía facilitó la adopción de medidas contra epidemias. En lo médico, el conocimiento de enfermedades recurrentes y mortales mejoró los tratamientos y, por último, el estudio de los cuerpos inertes mejoró la comprensión de las causas de la muerte.

\section{Las causas materiales de la mortalidad infantil}

Variadas conjeturas fueron establecidas para explicar las cifras altas de la mortalidad infantil en Santiago. La referida a los aspectos materiales de la sociedad se centró en la insalubridad urbana, en general, y en la insalubridad de las viviendas y el estado de los hospitales, en particular.

Los nacimientos en los conventillos, conjuntos habitacionales pobres pensados como "inmundas mazmorras" (Mortandad..., 2 dic. 1904, p.1), determinaban toda la trayectoria de la vida. El médico Enrique Deformes (30 nov. 1911, p.5) criticó la pésima calidad de esas construcciones sin ventilación, luminosidad, agua potable y alcantarillado. En esas "pocilgas" - escribió - "se siente el olor infecto producido por la acumulación de las inmundicias, por el desprendimiento de los vapores que brotan del suelo".

Juicios médicos similares catalogaron los conventillos de indignos incluso para comunidades salvajes (Sierra, Moore, 1895, p.9), al paso responsabilizaron al aire de las numerosas muertes de niños quienes eran los más perjudicados por ese "bárbaro sistema de habitación" (p.11). El médico Ángel Sanhueza sostuvo la necesidad de vivir en un medio con aire puro y fresco, disponiendo durante las noches, por lo menos, de veinticinco metros cúbicos para una buena oxigenación sanguínea. Atacaba, además, la "acción perniciosa de la habitación insalubre [que] se hace sentir especialmente en los niños" (Sanhueza, 24 feb. 1917, p.13).

Para el ingeniero Ismael Valdés Valdés (1918, p.269) apremiaba higienizarlos puesto que estaba demostrada su influencia en la salud y moral de los niños. Se debía optar por un modelo habitacional que tuviese amplitud "en vez de acumularse en una sola pieza [con los] animales". El temor de la elite a estas formas de vida guardaba relación con la alta tasa de fallecimientos prematuros, pero también con la promiscuidad entre humanos y animales "en medio de una contaminación total, aniquiladora del cuerpo" (Faltan..., 2 nov. 1906, p.5). De modo similar, la médica Cristina Aburto (1914, p.40) describió la convivencia de grupos familiares numerosos donde las enfermedades se reproducían rápidamente, como la viruela, perjudicando a los niños quienes "por su delicada constitución son incapaces de resistir". Esa enfermedad no se declaraba en las actas de defunción para evitar la desinfección de las habitaciones.

El aumento de la población inmigrante hacia Santiago demostró la precaria infraestructura habitacional y con ello la improvisación de medidas al tiempo que la mortalidad infantil ascendía (Altamirano, 1895; Urmeneta, 1984; De Ramón, 1990). Uno de los factores eran las 
acequias - conocidas localmente como "Ganges" en alusión al río de la India - para arrojar desechos orgánicos. Durante las epidemias, se rebasaban inundando calles y habitaciones con "desperdicios asquerosos" (Las acequias..., 29 sept. 1900, p.1). En los conventillos, "el agua cenagosa entra a las habitaciones o se empoza en los patios mal conformados" (p.1).

Ante estos hechos, en 1902, el Consejo Superior de Higiene Pública envió al Ministerio del Interior una nota describiendo los problemas de la red de acequias. La obra presentaba paredes defectuosas que permitían la filtración de excremento "que infesta el suelo de la ciudad" (Alcantarillado..., 30 dic. 1902, p.1). A ese problema se agregaba una corriente fluvial lenta que favorecía el depósito de "légamo putrescible" (p.1). Los médicos Sierra y Moore $(1895$, p.8) notaron la urgencia de sustituir el sistema de acequias por un moderno servicio de alcantarillas el que disminuiría el número de muertos y el deterioro de la salud infantil.

Los hospitales también presentaron problemas. En la mayoría de los casos, esos lugares atendieron a enfermos agonizantes. Los tratamientos y operaciones, debido a la aparición de infecciones, en casi la generalidad de los casos, concluían en la muerte. Los establecimientos médicos de beneficencia adolecían de materiales y especialistas para la atención de enfermos. La deficiencia económica de esos centros obligaba enviar a las comisarías de policía a quienes buscaban asistencia médica pública pues allí recibían "los más elementales cuidados" (La mortalidad..., 15 sept. 1916, p.10).

La pediatría tenía un escaso desarrollo, con lo cual las enfermedades infantiles no podían ser comprendidas en su complejidad. A esto se sumó la resistencia a la visita médica que ponían padres y menores. Una observación recurrente en los registros consideró “el pequeño número de niños que concurre a los hospitales, a causa, probablemente de no haber en estos establecimientos departamentos especiales para su asistencia" (Anuario..., 30 oct. 1874, p.1).

Las situaciones descritas impusieron la reorganización de las instituciones de saber médico. De ese modo se tuvo una mayor recepción de los avances médicos en un campo científico en conformación (La mortalidad..., 6 ene. 1908, p.3). En 1917, la Facultad de Medicina y Farmacia envió una carta al Ministerio del Interior proponiendo mejoras en el servicio de hospitales y la sustitución del reglamento orgánico que regía desde 1886. La facultad sugirió que "uno de los dos administradores del establecimiento fuese médico [ya que] los múltiples servicios necesitan ser organizados y vigilados por una persona que tenga la suficiente preparación técnica" (Organización..., 8 ago. 1917, p.12). Tales progresos dieron cuenta cómo desde la comunidad médica se estaba modificando la finalidad de los hospitales que, aparte de asilar, debían sanar a los pacientes para regresarlos sanos a la sociedad.

\section{Las causas morales de la mortalidad infantil}

Las conjeturas de orden moral para explicar la mortalidad infantil tuvieron una presencia importante en el discurso médico. Una primera característica de éstas fue su relación con la legitimidad o ilegitimidad al nacer. En rigor, desde 1884, el requisito para ser hijo legítimo era haber nacido dentro de un matrimonio civil y eclesiástico. En los hechos, el 50\% de los casos de mortalidad infantil correspondían a la categoría ilegítimo, ${ }^{10}$ situación que permitió 
al médico Federico Puga (1 feb. 1909, p.1) opinar en los siguientes términos: "si hubiera de juzgarse ... la inmoralidad de un país por la cantidad de sus hijos ilegítimos, ningún país habría más inmoral que Chile".

Durante el Congreso de Protección a la Infancia de 1912, el médico Víctor Körner (1913, p.138) comentó la diferencia de mortalidad entre niños legítimos e ilegítimos, la que en el caso de los últimos era "mucho mayor". Aburto $(1914$, p.13) iba más allá: "el niño ilegítimo que en nuestra clase baja es un ser generalmente engendrado bajo la influencia del alcohol, al nacer, desde luego, carece de padre, y la madre, que tampoco ha cedido por amor, en muchísimos casos abandona este hijo que para ella representa una pesada carga". ${ }^{11}$ Para la médica, además, la pertenencia al pueblo era sinónimo de ignorancia, alcoholismo, degradación física y criminalidad.

Una segunda característica de las causas morales fue su asociación - sobre todo en la prensa - con el alcoholismo. Por ejemplo una nota aseveró: "se sabe que la embriaguez se desarrolla i crece en proporción aterradora i es un hecho que la mortalidad de niños pobres es una de las mayores del mundo" (La enseñanza..., 22 feb. 1895, p.1). Para los medios, los padres alcohólicos tenían niños raquíticos y débiles o, dicho de otro modo, los vicios hacia esas bebidas causaban "los partos prematuros ... de estos niños débiles" (Melfi Demarco, 1907, p.39). Otro articulista enfatizó la diferencia entre los niños pobres y ricos (Mortalidad..., 13 nov. 1862, p.3). Por último, el alcohólico era pensado desde la elite periodística como un dilapidador de recursos, destructor de la familia, delincuente potencial y culpable de la degeneración racial. En síntesis, la mortalidad infantil fue pensada en términos de enfermedad social vinculada a otra de carácter epidemiológico. Prensa, médicos y políticos estigmatizaron a los pobres atacando el campo sexual. Los segundos intentaron explicar la baja tasa de muertes de niños causadas por la sífilis debido al ocultamiento de la "deshonrosa" enfermedad realizada por las madres. Se cerraba así el círculo de la culpabilidad: el ethos de hombres y mujeres populares sostenía la alta mortalidad infantil santiaguina.

\section{Las causas culturales de la mortalidad infantil}

Algunos especialistas criticaron con dureza las prácticas culturales maternas relacionadas con la alimentación y la higiene infantil. En el esquema patriarcal dominante en el Santiago del cambio de siglo, la vigilancia de los niños era tarea de mujeres, puesto que los padres pensaban que ello era actividad femenina (Sierra, Moore, 1895, p.18). La médica Aburto (1914, p.18) se oponía, por ejemplo, a la sustitución de la leche materna por otros alimentos que dañaban los órganos intestinales de los neonatos, "por la absoluta ignorancia de las madres". Por entonces era costumbre reemplazar la leche materna por alimentos sustanciosos como los caldos, ulpo y legumbres que, supuestamente, "fortificarían el organismo" (Algunas..., 1867, p.20).

Por otro lado, las vestimentas infantiles de invierno eran insuficientes. Desde la cultura se mantuvo la idea que a los niños era mejor acostumbrarlos desde pequeños al frío (Sierra, Moore, 1895, p.14). ${ }^{12}$ ¿Conviene abrigar a los niños? se preguntó un periódico a finales de los años 1880 reafirmando la popular idea que el poco abrigo fortalece a los niños porque disminuía los catarros y las pulmonías (Higiene..., 23 mar. 1889, p.1). 
Ante ambas situaciones relacionales entre padres y niños - alimentación y cuidados - el médico Enrique Deformes (30 nov. 1911, p.5) acusó el desconocimiento sobre higiene en las madres, especialmente en las relacionadas con la crianza "causa esencial de un gran número de afecciones graves que producen la muerte del recién nacido". Esta ignorancia, agregó, originaba otros hechos que ponían en riesgo a los menores: entrega a nodrizas, alimentación artificial, resistencia a las vacunas, descuido ante afecciones contagiosas e indiferencia de los padres ante la enfermedad. Ricardo Dávila (1899, p.345), por su parte, cuestionó la pobre atención dedicada por aquellos a los niños, recordando que "nunca se les ocurre siquiera echarlos a la cama".

Los curanderos y sus remedios también fueron responsabilizados de la alta mortalidad infantil. Los modos de pensar la enfermedad y la salud se demorarían décadas en modificarse culturalmente. El pueblo santiaguino prefirió asistir donde esos especialistas antes que visitar médicos profesionales. Cuando las enfermedades eran demasiado persistentes y arriesgaban la vida de la persona se acudía a los hospitales.

La curandería fue ejercida por conocedores de las tradiciones atávicas y de la magia, y contó con la reprobación de los médicos quienes se opusieron a ese conocimiento seudocientífico. Además, los acusados de "hechicería" - personas ignorantes, inhumanas y aprovechadoras del pueblo - disputaban a los científicos su campo profesional. A esto se sumó un desconocimiento y miedo de la población hacia los tratamientos médicos para niños, relacionado con las dificultades para "acertar con el diagnóstico en las enfermedades de los párvulos; pues se opone a ello la imposibilidad de hacer un examen prolijo, por el miedo que ellos tienen al galeno, [a quien] le falta poder informarse por boca del mismo del lugar de las dolencias" (Algunas..., 1867, p.22). Algunos padres no llevaban sus niños a las consultas médicas, diagnosticando el especialista en base a lo descrito por los adultos.

\section{Construir y legislar para la salud infantil}

La élite médica, política y periodística señalaba que el estancamiento demográfico de la ciudad tenía sus causas en la mortalidad infantil. Por ello comenzó a desarrollarse un discurso razonado sobre la necesidad de un hospital exclusivamente para niño, pues el Hospital San Juan de Dios solo contaba con setenta camas para este tipo de pacientes. Una experiencia pionera había sido efectuada por la Sociedad Protectora de la Infancia, la cual permitió que en 1899 las muertes infantiles disminuyesen en trescientas. Esa cifra era "un poderoso argumento a favor del hospital de niños" (Hospital..., 13 sept. 1900, p.2). Para los médicos "la única manera real y eficaz de combatir la horrenda mortalidad de párvulos ... es la fundación de ese hospital" (Dávila, 7 oct. 1900, p.1).

Seis años después un comité de señoras planteó el objetivo de construir el primer hospital de niños del país. Su tarea inicial fue constituir una comisión para colectar fondos entre la clase alta y el comercio. Además solicitaron apoyo parlamentario para obtener respaldo del gobierno. De gran necesidad fue conseguir la concesión de un terreno donde instalar el recinto, así como más recursos (El hospital..., 13 mayo 1905, p.5). Paralelamente, se pensó que el traslado del manicomio a un edificio nuevo solucionaría la falta de espacio para la 
atención infantil. El lugar tenía ventilación y se vería favorecido por "grandes patios, buen aire y mucho sol" (Hospital..., 13 sept. 1900, p.2).

Finalmente, en 1912 se construyó un hospital de niños, con los fondos legados por Manuel Arriarán, en la calle Santa Rosa sobre una antigua propiedad de Eduardo Matte adquirida por la Junta de Beneficencia de Santiago. Fue administrado por el expresidente Germán Riesco, secundado por los médicos jefes Alejandro del Río y Alfredo Commentz. La especialización en Europa de los últimos hizo pensar que el centro médico sería "uno de los mejores de la capital [y llenaría] una necesidad ... reclamada en Santiago" (El nuevo..., 13 nov. 1912, p.1). Como lo describió un diario de esa capital, el hospital de niños era de material sólido con "salas amplias, bien ventiladas y con bastante luz" (p.1), incluyendo parques y jardines que atraerían a los niños que ingresasen para tratarse (Commentz, 1918). ${ }^{13}$

Los proyectos y discusiones legislativas para disminuir las causas individuales de la mortalidad se extendieron por décadas, acusando la esterilidad de la clase política en la materia. Solo en 1904 comenzaron los debates de una ley que salvaguardase la niñez. El tema de la infancia se había transformado en materia política. Las posiciones giraron en torno al castigo con penas de cárcel por el abuso de niños, la obligación al trabajo forzado, la inducción a la mendicidad y la explotación. Los niños, como sujeto social, gozarían en teoría de la protección legal del Estado. No obstante, se debe destacar que la discusión se centró en el trabajo infantil, no considerando la mortalidad de los menores de un año. Todo esto demostró una valorización hacia la vida infantil, su educación formal y una protección para, como se intituló la ley, la infancia desvalida (Chile, 1912).

\section{Consideraciones finales}

Esta investigación analizó históricamente la mortalidad infantil, fenómeno inscrito en la denominada "cuestión social" chilena y que para superarla, durante 1860 y 1914, produjo una serie de discursos sociales sobre sus causas y estrategias.

El proceso de construcción de una medicina dedicada a la infancia se ha explicado como parte de la elaboración de conocimientos, entre los que participaron múltiples figuras, por ejemplo, los médicos, los curanderos, las mujeres y los infantes, así como un amplio espectro de argumentos que circularon sobre estos saberes. De ese modo, los fundamentos históricos de la pediatría no se pueden explicar solo por una visión de los doctores, sino que adquiere un significado más extenso; expresa las discusiones y los intercambios de argumentos sobre los tratamientos a los pequeños pacientes.

En el caso de la mortalidad infantil, la finalidad de esta investigación no pretende agotar un espacio de trabajo en el país, más bien trata de instalar nuevas interrogantes sobre esta temática, entre ellas: la constitución de una estadística uniforme y una nomenclatura sobre la niñez, los vínculos entre la maternidad-infancia y las transformaciones urbanas que mejoraron las condiciones materiales, entre otras temáticas. Nuestro análisis estuvo centrado en el conocimiento médico y su proyección a través de la prensa. El estudio se propuso describir detalladamente el fenómeno en base a publicaciones periódicas que observaron la mortalidad infantil con criterios moralistas. La elite productora de esas ideas 
se asignó la tarea de modificar las costumbres populares que en su lectura de los hechos fueron principales causantes de la catástrofe demográfica.

Las estadísticas, tanto eclesiásticas como civiles, a lo largo del siglo XIX y durante las primeras décadas del XX, atraviesan un proceso de mejoramiento en la recolección de los registros, procesamiento de la información y clasificación de los datos. A pesar de los errores, las críticas y las omisiones en las cifras, los argumentos de los facultativos, los médicos y publicaciones en la prensa coinciden en que Chile presenta una situación desconsoladora, siendo uno de los países con las más elevadas tasas de mortalidad a nivel mundial. Esta situación se modifica a inicios del siglo XX, con la aplicación de la clasificación de Bertillón; su contribución permite clarificar las causas de muerte, por lo tanto, mejorar los tratamientos y legitimar la instalación de una nueva especialidad médica dedicada a la infancia.

La medicina moderna, sostenida en los principios binarios de las costumbres civilizadas o barbáricas, construyó un discurso científico sesgado contra el pueblo. Según su argumentación, los menores fallecían por su condición de niños ilegítimos, por el alcoholismo de sus padres y por los malos cuidados de sus madres, entre otros problemas relacionados con la alimentación y el cuidado en general durante los primeros años de vida. Con este saber se reafirmaba un tipo de familia tradicional, pero no se disminuía el indicador de mortalidad.

La mortalidad infantil dio cuenta de los conocimientos médicos de la época, dominados por el paradigma "atmosférico" que explicó comportamientos de la salud en función del clima. Otra característica de aquellos fue la disputa con los curanderos, la inexistencia de médicos especialistas en la salud de los niños y los temores populares hacia los tratamientos científicos.

La complejidad de la situación demográfica infantil hizo que las autoridades políticas debiesen encarar el problema con nuevas fórmulas. De ese modo se pensó que la educación de los niños del pueblo, a través de la escuela, sería una alternativa viable. Lo mismo con el apoyo de la prensa, medio de comunicación proclive a formar opiniones públicas referidas al cuidado de la salud de los más pequeños. Dos grandes avances se lograron al finalizar la etapa de estudio: la creación de la ley sobre protección a la infancia desvalida (1912) y la inauguración del Hospital Manuel Arriarán (1913).

Para finalizar, se debe considerar que la construcción de un problema historiográfico se encuentra en configuración permanente, por lo que la búsqueda de nuevas interrogantes y líneas de análisis resultan fundamentales. Esto conlleva que las propuestas de trabajo debiesen tener en cuenta las discusiones y las definiciones sobre lo que se requiere para visualizar las situaciones que incidieron en la infancia en el cambio de siglo XIX-XX, lo que abriría campos de estudio y vinculaciones con otras disciplinas, tanto a escala local como regional.

\section{AGRADECIMIENTOS}

Esta investigación ha sido elaborada con el financiamiento otorgado por la Comisión Nacional de Investigación Científica y Tecnológica (Conicyt), a través de su programa formación de capital humano avanzado: Beca de Doctorado Nacional (2015) y Beca de Doctorado en el Extranjero “Becas Chile” (2016). 


\section{NOTAS}

${ }^{1}$ La estadística permitió expresar la magnitud del problema y diferenciar la incidencia del fenómeno según grupos etarios: la ocurrida desde el nacimiento hasta el primer año de vida y la desarrollada entre el primer año y los cuatro años. De igual modo, la clasificación según las causas de las enfermedades podían ser exógenas, tales como infecciones, alimentación inadecuada y accidentes o endógenas, por ejemplo, dificultades del parto, malformaciones o debilidad congénita. Estas situaciones han sido abordadas en España (Arrizabalaga, 1993; Miccó, Martínez, 1993; Perdiguero, 1993), en Francia (Bourgeois, 1951; Preston, 1975, 1980; Knodel, 1983) y en Argentina (Celton, 1992; Di Liscia, 2002; Mazzeo, 2007).

${ }^{2}$ Las cifras de mortalidad infantil (0 a 7 años) entregadas en el Anuario estadístico de 1862 fueron las siguientes: Obispado de Concepción: 4.985 niños fallecidos; Arzobispado de Santiago: 15.239; Obispado de La Serena: 1.892 (Oficina..., 1862). Según el Anuario estadístico de 1879 las cifras en los Departamentos fueron: Concepción 499, Santiago 5.042 y La Serena 825 (Oficina..., 1879). En 1909, una publicación de prensa recogió las estadísticas entregadas por el Registro Civil, señalando que en 1908 se produjeron en Chillán 1.142 niños muertos entre los 0 a 7 años (La mortalidad..., 30 ene. 1909, p.1). De igual modo, Eduardo Cordero Baños en Los males sociales como causa de la mortalidad infantil, aludiendo al Anuario estadístico, señaló que la cantidad de niños muertos antes de un año, durante el año 1915, fue: en Tarapacá 747 fallecidos, en Santiago 7.193 y en Arauco 642.

${ }^{3}$ Según el Anuario estadístico las cifras de población en el Departamento de Santiago fueron las siguientes: 208.708 habitantes (año 1879), 205.269 (1882), 488.382 (1917), 564.873 (1921), 587.887 (1923), 599.390 (1924), 610.901 (1925), 622.408 (1926), 645.638 (1927), 739.492 (1928), 736.736 (1929) y 839.565 (1930).

${ }^{4}$ Agregamos, las caracterizaciones, en diversos espacios geográficos, desde lo moral, la justicia y el infanticidio (Guimarães et al., 2003; Olivieri, 2010; Díaz, Gallego, Lafortune, 2016).

${ }^{5}$ La creación de una institucionalidad laica produjo colisiones. Andrés Irarrázaval estableció una continuidad entre los registros eclesiásticos y civiles al comparar las partidas con los certificados de bautismo, matrimonio y defunción. Demostró que ambos recogieron datos similares y mantuvieron vigencia jurídica (Irarrázaval, 2014, p.315-341). El Registro Civil recibió críticas sobre su funcionamiento ralentizado, la escasez de empleados y los bajos sueldos de los mismos. Ver: La constitución... (19 ene. 1920, p.16).

${ }^{6}$ Según Isauro Torres (1918, p.11), los servicios estadísticos chilenos eran deficientes, siendo fácil encontrar datos distintos en los boletines de la Oficina Central de Estadística o en las Memorias del Ministerio de Justicia, aun cuando compartían la fuente: el Registro Civil.

${ }^{7}$ La estadística de sepultaciones de 1904 consideró las siguientes cifras (por miles de habitantes): Montevideo $(16,2)$, Berlín, La Haya, Turín $(17,1)$, Buenos Aires $(17,7)$, Estocolmo $(17,9)$, Roma $(18,4)$, Londres $(18,9)$, Ginebra (19), Edimburgo (19,5), Río de Janeiro (19,6), Nueva York (19,7), París $(19,8)$, Viena (20), Burdeos y Budapest (21), Manchester (21,8), Liverpool (23,7), San Petersburgo (25,5), Dublín $(26,6)$, Nápoles $(29,6)$, Madrid (29,8), Cracovia $(32,4)$, Concepción (41), Valparaíso $(41,7)$, Pernambuco $(42,1)$ y Santiago $(48,7)$ (Mortalidad, 7 ene. 1905, p.8).

${ }^{8}$ Para argumentar esta frase mostraba un cuadro de mortalidad de las principales ciudades del globo en relación con su población en 1894. Entre las ciudades con menores índices de defunciones por cada mil habitantes estaban: La Haya $(16,9)$, Berlín $(17,2)$, Londres $(17,7)$, Edimburgo $(17,8)$, Bruselas $(18,1)$ y Leipzig $(18,7)$. Los centros urbanos con mayores cifras eran: Santiago de Chile $(46,14)$, Alejandría $(49,8)$, Bombay $(52,9)$ y El Cairo $(53,4)$ (Murillo, 1896, p.11).

${ }^{9}$ Enmarcada en el sistema de división política-administrativa de Chile durante el siglo XIX, la Provincia de Santiago surge con las leyes federales (1826). Posteriormente, la Constitución de 1833 estableció su dirección bajo la figura de un Intendente. Estas se jerarquizaban en departamentos, subdelegaciones y distritos.

${ }^{10}$ El Chileno publicó cifras sobre este problema con la intención, quizás, de resguardar la moralidad pública y magnificar los hechos. Algunos índices fueron: en Santiago 49.7\%, en Viena 31\%, en París 26.6\%, en Berlín, Florencia y Roma 16\%, en Buenos Aires 14\%. Comparativamente, las cifras son discutibles debido a que cada país legisló de modo diferente la ilegitimidad de los nacidos. En Chile, el Registro Civil catalogó de ilegítimas todas las familias no casadas por la ley. Estos números resultan discutibles al ser vistos comparativamente, dado que la ilegitimidad al tener un componente legal, permite que cada país construya una definición específica en la materia.

${ }^{11}$ Por su lado, el médico Ricardo Dávila (7 oct. 1900, p.1), sostuvo que "las tres cuartas partes de los niños perecen hoy por el abandono miserable que se ven en sus enfermedades". 
${ }^{12}$ En el mismo estudio, los doctores señalan que el pueblo tiene ideas coloniales de higiene que mezcladas con las araucanas daban como resultado, la creencia que la exposición al frío no dañaría los organismos de los niños.

${ }^{13}$ En esta publicación, el médico realizó una descripción detallada del recinto, sobre todo de la sección de cirugía. También entregó una serie de planos y croquis de los pabellones y su construcción.

\section{REFERENCIAS}

ABURTO, Cristina. Contribución al estudio de la mortalidad infantil en Santiago. Memoria para optar al grado de licenciado en medicina, Universidad de Chile. Santiago: Imprenta y Litografía La Ilustración. 1914.

ALCANTARILLADO...

Alcantarillado de Santiago. Las Últimas Noticias, p.1. 30 dic. 1902 .

\section{ALGUNAS...}

Algunas ideas sobre la excesiva mortalidad de los párvulos y medios profilácticos que podían emplearse. Revista el Médico Práctico, año 1, n.2, p.13-24. 1867.

ALTAMIRANO, Carlos.

Apuntes para un estudio sobre habitaciones obreros. Revista Chilena de Higiene, t.2, n.4, p.554. 1895.

ANGUITA, Ricardo.

Leyes promulgadas en Chile: desde 1810 hasta el 1 de junio de 1913. Santiago: Imprenta, Litografía y Encuadernación Barcelona. 1913.

ANUARIO ...

Anuario estadístico de Chile. El Ferrocarril, p.1. 30 oct. 1874.

APPLE, Rima.

Mothers and medicine: a social history of infant feeding, 1890-1950. Madison: The University of Wisconsin Press. 1987.

APPLE, Rima; GOLDEN, Janet.

Mothers and motherhood: readings in American

history. Columbus: Ohio State University. 1997.

ARBAIZA, Mercedes; GUERRERO, Ana; PAREJA, Arantza.

Mundo rural y mundo urbano en la transición de la mortalidad vizcaína, 1770-1930. Boletín de la Asociación de Demografía Histórica, v.14, p.1956. 1996.

ARMUS, Diego.

La ciudad impura: salud, tuberculosis y cultura en Buenos Aires, 1870-1950. Quinto Sol, n.13, p.205-236. 2009.

ARMUS, Diego.

La enfermedad en la historiografía de América

Latina moderna. Asclepio, v.54, n.2, p.41-60. 2002.
ARMUS, Diego.

Cuando los enfermos hacen huelga: Argentina, 1900-1940. Revista Estudios Sociales, n.20, p.5380. 2001.

ARRIZABALAGA, Jon.

La identificación de las causas de muerte en la Europa pre-industrial: algunas consideraciones historiográficas. Revista de Demografía Histórica, v.11, n.3, p.23-47. 1993.

BALLESTER, Rosa.

En torno al siglo de los niños. Dynamis, n.25, p.539-545. 2005.

BALLESTER, Rosa; BALANGUER, Emilio. La infancia como valor y como problema en las luchas sanitarias de principios de siglo en España. Dynamis, n.15, p.177-192. 1995.

BELLETINNI, Athos.

Evolution différentielle et mouvement saisonnier de la mortalité infantile et enfantine dans la banlieue de Bologne, XVIIIe-XIXe siècles. Annales de Démographie Historique, n.1, p.195-207. 1983.

BERNABEU, José.

Expresiones diagnósticas y causas de muerte: algunas reflexiones sobre su utilización en el análisis demográfico de la mortalidad. Revista de Demografía Histórica, v.11, n.3, p.11-21. 1993.

BERNABEU, José et al.

El análisis histórico de la mortalidad por causas: problemas y soluciones. Revista de Demografía Histórica, v.21, n.1, p.167-193. 2003.

BERNABEU, José; LÓPEZ, José María.

Condicionantes de la mortalidad entre $1800 \mathrm{y}$ 1930: higiene, salud y medio ambiente. Boletín de la Asociación de Demografía Histórica, v.5, p.7079. 1987.

BIRN, Anne-Emanuelle.

Doctors on record: Uruguay's infant mortality stagnation and its remedies, 1895-1945.

Bulletin of the History of Medicine, n.82, p.311354. 2008.

BIRN, Anne-Emanuelle.

Child health in Latin America: historiographic perspectives and challenges. História, Ciências, Saúde-Manguinhos, v.14, n.3, p.677-708. 2007. 
BIRN, Anne-Emanuelle.

No se debe llorar sobre la leche derramada: el pensamiento epidemiológico y la mortalidad infantil en Uruguay, 1900-1940. Estudios Interdisciplinarios de América Latina y el Caribe, v.14, n.1, p.35-65. 2003.

BOURGEOIS, Jean.

La mesure de la mortalité infantile: principes et méthodes. Population, n.2, p.233-248. 1951.

CALVO MACKENNA, Luis.

El Patronato Nacional de la Infancia en la actualidad. Revista de Beneficencia Pública, t.3, n.2, p.24-43. 1919.

CAVIERES FIGUEROA, Eduardo.

Ser infante en el pasado: triunfo de la vida o persistencia de estructuras sociales: la mortalidad infantil en Valparaíso, 1880-1950. Revista de Historia Social y de las Mentalidades, n.5, p.31-58. 2001.

CELTON, Dora.

La mortalidad en la ciudad de Córdoba (Argentina) entre 1869 y 1990. Boletín de la Asociación de Demografía Histórica, v.10, n.1, p.31-57. 1992.

\section{CHAUNU, Pierre.}

Historia y población: un futuro sin porvenir.

México: Fondo de Cultura Económica. 1982.

CHILE.

Ministerio de Justicia. Ley n.2.765 sobre protección a la infancia desvalida. Boletín de las Leyes i Decretos del Gobierno, libro 81. Santiago: Imprenta Nacional. 1912.

CHILE.

Ministerio de Justicia, Culto e Instrucción Pública. Ley del Registro Civil, n.2.184, promulgada en 16 jul. 1884. Boletín de las Leyes i Decretos del Gobierno. Santiago: Imprenta Nacional. 1884.

CHILE...

Chile en la estadística internacional. El Mercurio, p.7. 18 sept. 1908.

COMMENTZ, Alfredo.

El Hospital de Niños Manuel Arriarán, Santiago. Revista de Beneficencia Pública, t.2, n.2, p.154164. 1918.

CROIZET, Emilio.

Lucha social contra la mortalidad infantil en el período de lactancia: conferencia dada en la Universidad de Chile el 7 de septiembre de 1912. Santiago: Imprenta, Litografía y Encuadernación Barcelona. 1913.

DÁVILA, Ricardo.

Obra nacional: hospitales para niños. El Chileno, p.1. 7 oct. 1900 .
DÁVILA, Ricardo.

Mortalidad de los niños en Santiago: sus causas y sus remedios. Revista Chilena de Higiene, t.5, n.8, p.277-294. 1899.

DEFORMES, Enrique.

Las Últimas Noticias, p.5. 30 nov. 1911.

DELGADO, Manuel.

La infancia abandonada en Chile, 1770-1930.

Revista de Historia Social y de las Mentalidades, n.5, p.101-126. 2001.

DEL RÍO, Alejandro.

[Tablas con la mortalidad de infantes]. Boletín de Higiene y Demografía, t.1-14. 1898-1911.

DE RAMÓN, Armando.

La población informal: poblamiento de la periferia de Santiago de Chile, 1920-1970. Revista Eure, v.16, n.50, p.5-17. 1990.

DÍAZ, Eloísa.

La alimentación de los niños pobres en las escuelas públicas: informe presentado al señor ministro de Instrucción Pública por el Médico Inspector de Escuelas de Santiago. Santiago: Imprenta, Litografía y Encuadernación Barcelona. 1906.

DÍAZ, José; GALLEGO, Francisco; LAFORTUNE, Jeanne.

Nacimientos fuera del matrimonio en la historia de Chile: algunos hechos estilizados. Estudios Públicos, n.142, p.37-79. 2016.

DI LISCIA, María Silvia.

Hijos sanos y legítimos: sobre matrimonio y asistencia social en Argentina, 1935-1948.

História, Ciências, Saúde - Manguinhos, v.9, supl., p.209-232. 2002.

EHRICK, Christine.

Mothers and the colossal machine: feminism, social assistance and the State in Uruguay, 19101932. The Americas, v.58, n.1, p.121-139. 2001.

EL HOSPITAL...

El hospital para niños. El Mercurio, p.5. 13 mayo 1905.

EL NUEVO...

El nuevo Hospital de Niños. Las Últimas Noticias, p.1. 13 nov. 1912.

ESTEFANE, Andrés.

La institucionalización del sistema estadístico chileno: debates y problemas prácticos, 18431851. Estudios Sociales del Estado, v.2, n.4, p.3573. 2016.

ESTEFANE, Andrés.

Burócratas ambulantes: movilidad y producción de conocimiento estadístico en Chile, 18601873. Revista Enfoques, v.11, n.17, p.123-146. 2012. 
ESTEFANE, Andrés.

Un alto en el camino para saber cuántos somos... Los censos de población y la construcción de lealtades nacionales (Chile, siglo XIX). Revista História, v.1, n.1, p.33-59. 2004.

FALTAN...

Faltan estadísticas. El Mercurio, p.5. 2 nov. 1906.

FARIÑAS, Diego; SANZ GIMENO, Alberto. Cambios estructurales en la mortalidad infantil y juvenil española, 1860-1990. Boletín de la Asociación de Demografía Histórica, v.17, n.1, p.49. 87. 1999.

FREIRE, Maria Martha de Luna.

A construção da "maternidade científica" no Brasil republicano: mulheres, mães e médicos: discurso maternalista no Brasil. Estudos Feministas, v.18, n.3, p.941-964. 2010.

FREIRE, Maria Martha de Luna.

"Ser mãe é uma ciência": mulheres, médicos e a construção da maternidade científica na década de 1920. História, Ciências, Saúde - Manguinhos, v.15, supl., p.153-171. 2008.

GALLO, Óscar; MÁRQUEZ, Jorge.

La mortalidad infantil y la medicalización de la infancia: el caso de Titiribí, Antioquía, 1910-1950. Revista Historia y Sociedad, n.20, p.57-89. 2011.

GARCÍA, Carlos.

Niños trabajadores y vida cotidiana en Medellín, 1900-1930. Medellín: Editorial Universidad de Antioquía. 1999.

GARCÍA, Juan César.

La medicina estatal en América Latina, 18801930. Revista Cubana de Salud Pública, v.42, n.1, p.143-175. 2016.

GOICOVIC, Igor.

Sociabilidad de los niños y jóvenes populares en el Chile tradicional. In: Sagredo, Rafael; Gazmuri, Cristian (Dir.). Historia de la vida privada en Chile, t.1: el Chile tradicional de la conquista a 1840. Santiago: Aguilar Chilena; Taurus. p.231-267. 2005.

GREZ TOSO, Sergio.

La cuestión social en Chile: ideas y debates precursores, 1804-1902. Santiago: Dibam. 1997.

GUIMARÃES, Maria José Bezerra et al. Condição de vida e mortalidade infantil: diferenciais intra-urbanos no Recife, Pernambuco, Brasil. Cadernos de Saúde Pública, v.19, n.5, p.1413-1424. 2003.

HIGIENE...

Higiene en la infancia ¿Conviene abrigar a los niños? El Chileno, p.1. 23 mar. 1889.
HOSPITAL...

Hospital de niños: urgencia de establecerlo. $E l$ Mercurio, p.2. 13 sept. 1900.

ILLANES, María Angélica.

En el nombre del pueblo, del Estado y de la ciencia: historia social de la salud pública, Chile, 18801973: hacia una historia social del Siglo XX. Santiago: Colectivo de Atención Primaria. 1993.

ILLANES, María Angélica.

'Ausente, señorita': el niño chileno, la escuela para pobres y el Auxilio, 1890-1990. Santiago: Junta Nacional de Auxilio Escolar y Becas. 1991.

IRARRÁZAVAL, Andrés.

Los inicios del registro civil de Chile: ¿Ruptura o continuidad con las antiguas partidas eclesiásticas? Revista de Estudios Jurídicos, n.36, p.315-341. 2014.

KNODEL, John.

Seasonal variation in infant mortality: an approach with applications. Annales de Démographie Historique, n.1, p.208-230. 1983.

KÖRNER, Emilio et al.

Primer Congreso Nacional de Protección a la Infancia: trabajos y actas. Santiago: Imprenta Barcelona. 1913

LA CONSTITUCIÓN...

La constitución de la familia: errores funestos que es necesario corregir. El Mercurio, p.16. 19 ene. 1920.

LA ENSEÑANZA...

La enseñanza de la higiene. El Chileno, p.1. 22 feb. 1895.

LA MORTALIDAD...

La mortalidad de los párvulos en Chile. Revista Médica de Chile, año 3, n.9, p.340-341. mar. 1875.

LA MORTALIDAD...

La mortalidad en Chillán: su estadística en 1908. El Chileno, p.1. 30 ene. 1909.

LA MORTALIDAD...

La mortalidad en Santiago. El Mercurio, p.10. 15 sep. 1916.

LA MORTALIDAD...

La mortalidad en Santiago. El Mercurio, p.3. 6 ene. 1908.

LA MORTANDAD...

La mortandad en la niñez. El Mercurio, p.6. 29 dic. 1902.

LAS ACEQUIAS...

Las acequias de Santiago. El Chileno, p.1. 29 sept. 1900.

LEBRUN, François.

"La mortalité des enfants dans le monde et dans l'histoire", publié sous la direction de Paul-Marie 
Boulanger et Dominique Tabutin, 1980. Annales de Démographie Historique, n.1, p.389-391. 1982.

LINDÓN, Alicia; HIERNAUX, Daniel (Dir.). Tratado de geografía humana. México: Anthropos. 2006.

MASUY-STROOBANT, Godelieve.

La surmortalité infantile des Flandres aucours de la deuxième moitié du XIXe siècle: mode d'alimentation ou mode de développement? Annales de Démographie Historique, n.1, p.231256. 1983.

MAZZEO, Victoria.

La mortalidad de la primera infancia en la Ciudad de Buenos Aires en el período 1860-2002. Papeles de Población, v.13, n.53, p.241-272. 2007.

MELFI DEMARCO, Mateo.

Mortalidad infantil y protección a la infancia. Memoria (Para optar al grado de Licenciado) Facultad de Medicina y Farmacia, Universidad de Chile, Santiago. 1907.

MICCÓ, José Antonio; MARTÍNEZ, Francisco. La utilización de las técnicas del análisis semántico-documental en el estudio e interpretación de las expresiones diagnósticas de las causas de muerte. Revista de Demografía Histórica, v.11, n.3, p.173-186. 1993.

MILANICH, Nara.

Women, children, and domestic labor in nineteenth-century Chile. Hispanic American Historical Review, v.91, n.1, p.29-62. 2011.

MILANICH, Nara.

Children of fate: childhood, class, and the State in Chile, 1850-1930. Durham: Duke University Press. 2009.

\section{MILANICH, Nara.}

Casa de Huérfanos and child circulation in late-nineteenth century Chile. Journal of Social History, v.38, n.2, p.311-340. 2004.

MILANICH, Nara.

Los hijos de la providencia: el abandono como circulación en el Chile decimonónico. Revista de Historia Social y de las Mentalidades, n.5, p.79-100. 2001.

MILANICH, Nara.

Entrañas mil veces despreciables e indignas: el infanticidio en el Chile tradicional. Dimensión Histórica de Chile, n.13-14, p.63-82. 1998.

MORATA, Eva María.

La imagen de la maternidad en la España de finales del siglo XIX y principios del XX. Revista Arenal, v.10, n.2, p.163-190. 2003.

MORENO, José Luis.

El delgado hilo de la vida: los niños expósitos de
Buenos Aires, 1779-1823. Revista de Indias, v.60, n.220, p.663-685. 2000.

MORTALIDAD.

Mortalidad. El Mercurio, p.8. 7 ene. 1905.

MORTALIDAD...

Mortalidad de párvulos en Chile: datos

elocuentes. La Época, p.2. 18 dic. 1882.

MORTALIDAD...

Mortalidad de Santiago. El Ferrocarril, p.3.

13 nov. 1862 .

MORTANDAD...,

Mortandad de niños. El Diario Popular, p.1. 2 dic. 1904.

MOVIMIENTO...

Movimiento de población. El Ferrocarril, p.3. 7 jul. 1856.

MURILLO, Adolfo.

La mortalidad urbana en Chile: discurso leído en la sesión de apertura del Congreso Científico General Chileno celebrado en Concepción. Santiago: Imprenta y Encuadernación Roma. 1896.

OCUPAMOS...

Ocupamos un lugar muy superior de las ciudades que tienen mayor mortalidad en el orbe entero. El Mercurio, p.5. 7 ene. 1905.

OFICINA...

Oficina Central de Estadística. Anuario estadístico de la República de Chile. Santiago: Imprenta Nacional. 1879.

OFICINA...

Oficina Central de Estadística. Anuario estadístico de la República de Chile. Santiago: Imprenta Nacional. 1862.

OLIVIERI, Ilaria.

L'infanticidio tra percezione popolare e trattamento giuridico: Prignano, 1889.

Storicamente, n.6. Disponible en: <http://dx.doi. org/10.1473/stor71>. Acceso en: 12 jun. 2017. 2010.

ORGANIZACIÓN...

Organización de la Beneficencia Pública: opinión de la Facultad de Medicina. El Mercurio, p.12. 8 ago. 1917.

OSTA, María Laura.

Niños y niñas, expósitos y huérfanos en Montevideo del siglo XIX. Revista de la Facultad de Derecho, n.41, p.155-189. 2016.

PALMA, Patricia.

Mortalidad infantil en Chile: la situación médica social en la Casa de Expósitos de Santiago 1889-1899. Anales de Historia de la Medicina, v.19, n.2, p.127-137. 2009. 
PERDIGUERO, Enrique.

Causas de muerte y relación entre conocimiento científico y conocimiento popular. Revista de Demografía Histórica, v.11, n.3, p.65-88. 1993.

PITA, Valeria Silvina.

¿La ciencia o la cultura? Pujas entre médicos y matronas por el dominio institucional: Buenos Aires, 1880-1900. In: Álvarez, Adriana; Molinari, Irene; Reynoso, Daniel. Historias de enfermedades, salud y medicina en la Argentina de los siglos XIX$X X$. Mar del Plata: Universidad del Mar del Plata. 2004.

PRESTON, Samuel.

Causes and consequences of mortality declines in less developed countries during the twentieth century. In: Easterlin, Richard A. Population and economic change in developing countries. Chicago: University of Chicago Press. 1980.

PRESTON, Samuel.

The changing relation between mortality and level of economic development. Population Studies, v.29, n.2, p.231-248. 1975.

PUGA, Federico.

La mortalidad infantil: una causa primordial del daño: disolución de la familia e inmoralidades. El Chileno, p.1. 1 feb. 1909.

REVUELTA, Bárbara.

¿Qué pasa en La Inclusa? The role of press scandals, doctors and public authorities in the evolution of La Inclusa de Madrid, 1890-1935. Dynamis, n.35, p.107-130. 2015.

REVUELTA, Bárbara.

Abandoned and illegitimate, a double mortality penalty? Mortality of illegitimate infants in the foundling hospital of Madrid, La Inclusa (18901935). Journal of the History of the Family, v.18, n.1, p.44-67. 2013.

REVUELTA, Bárbara.

Releasing mother's burden: child abandonment and retrieval in the foundling hospital of Madrid, 1890-1935. Journal of Interdisciplinary History, v.42, n.4, p.645-672. 2012.

RODRÍGUEZ, Esteban.

La salud infantil, asunto ejemplar en la historiografía contemporánea. Dynamis, n.23, p.27-36. 2003.

RODRÍGUEZ, Esteban.

La construcción de la salud infantil: ciencia, medicina y educación en la transición sanitaria en España. Cuadernos de Historia Contemporánea, n.18, p.19-52. 1998.

ROJAS FLORES, Jorge.

Historia de la infancia en el Chile republicano, 1810-2010. Santiago: Junta Nacional de Jardines Infantiles. 2010.
ROJAS FLORES, Jorge.

Los suplementeros: los niños y la venta de diarios. Chile, 1880-1953. Santiago: Ariadna. 2006.

ROMERO, Luis Alberto.

¿Qué hacer con los pobres? Elites y sectores populares en Santiago de Chile, 1840-1895. Santiago: Ariadna. 2007.

ROMERO, Luis Alberto.

Condiciones de vida de los sectores populares en Santiago de Chile, 1840-1895 (vivienda y salud). Nueva Historia, n.9, p.5-86. 1984.

RUGGIERO, Kristin.

Maternity, and the disciplining of women: infanticide in late nineteenth-century Buenos Aires. The Hispanic American Historical Review, v.72, n.3, p.353-373. 1992.

RUSTOYBURU, Cecilia.

Maternidad e infancia: los consejos médicos y psicológicos en la revista "Hijo Mío...!", Buenos Aires, en la década de 1930. Revista Arenal, v.23, n.1, p.117-142. 2016.

RUSTOYBURU, Cecilia.

Pediatría psicosomática y medicalización de la infancia en Buenos Aires, 1940-1970. História, Ciências, Saúde-Manguinhos, v.22, n.4, p.12491265. 2015.

RUSTOYBURU, Cecilia.

Infancia, hormonas y género: un análisis histórico de los discursos de la biotipología en Argentina en los años 1930. Revista Latinoamericana: Sexualidad, Salud y Sociedad, n.11, p.9-36. 2012.

SALAZAR, Gabriel. Ser niño "huacho" en la historia de Chile (siglo $X I X)$. Santiago: LOM Ediciones. 2006.

SALAZAR, Gabriel.

Labradores, peones y proletarios: formación y crisis de la sociedad popular chilena del siglo XIX. Santiago: Ediciones Sur. 1985.

SALINAS, René.

Orphans and family disintegration in Chile: the mortality of abandoned children, 1750-1930. Journal of Family History, v.16, n.3, p.315-329. 1991.

SALINAS, René; DELGADO, Manuel.

Los hijos del vicio y del pecado: la mortalidad de los niños abandonados, 1750-1930. Proposiciones, n.19, p.44-54. 1990.

SÁNCHEZ, Jesús.

El crecimiento de la población mundial: implicaciones socioeconómicas, ecológicas y éticas. Valencia: Tirant lo Blanch. 2008.

SANHUEZA, Ángel.

El Mercurio, p.13. 24 feb. 1917. 
SANZ GIMENO, Alberto; FARIÑAS, Diego. La caída de la mortalidad en la infancia en la España interior, 1860-1960: un análisis de las causas de muerte. Cuadernos de Historia Contemporánea, v.24, n.1, p.151-188. 2002.

SATER, William.

The politics of public health: smallpox in Chile. Journal of Latin American Studies, v.35, n.3, p.513543. 2003.

SIERRA, Lucas; MOORE, Eduardo.

La mortalidad de los niños en Chile (publicación de la Sociedad Protectora de la Infancia). Valparaíso: Imprenta y Litografía Central. 1895.

SOMMERS, Carlos.

La mortalidad en Chile durante los años 1892, 1893, 1894 y 1895. Revista Chilena de Higiene, t.3, n.5, p.373-395. 1896.

TORRES, Isabel.

El imaginario de las elites y los sectores populares, 1919-1922. Santiago: Editorial Universitaria. 2011.

TORRES, Isabel.

Los conventillos en Santiago, 1900-1930.

Cuadernos de Historia, n.6, p.67-85. 1986.

TORRES, Isauro.

Mortinatalidad de Santiago (abortos y nacidos muertos). Santiago: Imprenta "El Progreso". 1918.

URMENETA, Roberto.

Condiciones físicas y sociales de conventillos, cités, pasajes y residenciales en la zona centro de
Santiago: análisis descriptivo. Tesis (Maestria en Estudios Urbanos) - Pontificia Universidad Católica de Chile, Santiago. 1984.

VALDÉS VALDÉS, Ismael.

El problema de la infancia. Revista de Beneficencia Pública, t.2, n.2, p.267-272. 1918.

ZÁRATE, Soledad.

Embarazo y amamantamiento: cuerpo y reproducción en Chile. In: Góngora, Álvaro; Sagredo, Rafael (Dir.). Fragmentos para una historia del cuerpo en Chile. Santiago: Taurus. 2011.

ZÁRATE, Soledad.

El licor de la vida: lactancia y alimentación materno-infantil en Chile, 1900-1950. In: Sciolla, Carolina (Comp.). Historia y cultura de la alimentación en Chile. Santiago: Catalonia. p.235261. 2010.

ZÁRATE, Soledad.

De partera a matrona: hacia la asistencia profesional del parto en Chile en el siglo XIX. Calidad en la Educación, n.27, p.284-297. 2007.

ZÁRATE, Soledad.

Parto, crianza y pobreza en Chile. In:

Sagredo, Rafael;Gazmuri, Cristian (Dir.). Historia de la vida privada en Chile, t.3: el Chile contemporáneo de 1925 a nuestros días. Santiago: Aguilar Chilena; Taurus, p.13-47. 2005. 